\title{
Efficacy and safety of endoscopic mucosal resection of non-ampullary duodenal polyps: a systematic review
}

Authors

Institutions
Udayakumar Navaneethan ${ }^{1}$, Muhammad K. Hasan' ${ }^{1}$, Vennisvasanth Lourdusamy ${ }^{1,2}$, Xiang Zhu' ${ }^{1}$, Robert H. Hawes ${ }^{1}$, Shyam Varadarajulu'

${ }^{1}$ Center for Interventional Endoscopy, Orlando, FL, USA

2 Department of Internal Medicine, Brandon Regional Hospital, Brandon, FL, USA submitted: 7. January 2016 accepted after revision: 11. April 2016

\section{Bibliography} Dol http://dx.doi.org/ 10.1055/s-0042-107069 Endoscopy International Open 2016; 04: E699-E708 (c) Georg Thieme Verlag KG Stuttgart · New York E-ISSN 2196-9736

\section{Corresponding author} Udayakumar Navaneethan, MD

Center for Interventional Endoscopy University of Central Florida College of Medicine Florida Hospital 601 E Rollins Street Orlando

FL 32814

USA

Fax: +1-407-303-2585 udhaykumar81@gmail.com
Background and aims: Data on the safety and efficacy of endoscopic resection of non-ampullary duodenal polyps are limited. This study evaluated the safety and efficacy of endoscopic mucosal resection (EMR) of sporadic non-ampullary duodenal polyps.

Methods: Relevant studies for the meta-analysis were identified through search of PUBMED and EMBASE databases. Studies employing EMR for the management of sporadic duodenal polyps in the non-ampullary region were included. The primary outcome was the surgical intervention rates due to non-curative endoscopic resection (incomplete removal/recurrence necessitating surgery) and/or management of procedural adverse events.

Results: A total of 440 patients (485 duodenal polyps) from 14 studies were included. The mean size of the polyps was $13 \mathrm{~mm}$ to $35 \mathrm{~mm}$. Surgical intervention due to non-curative EMR

\section{Introduction}

\section{$\nabla$}

Sporadic duodenal polyps are rare tumors of the gastrointestinal tract, often diagnosed incidentally during an upper endoscopy [1]. Duodenal tumors/polyps encompass a variety of pathologies of which adenoma is most commonly encountered [2]. Adenomas in the duodenum follow the adenoma-carcinoma sequence similar to colonic adenomas [3]. Their removal is necessary owing to their potential for malignant transformation.

Traditionally, duodenal polyps have been managed surgically; however, surgical resection of these polyps is often associated with increased morbidity when compared to endoscopy [4], as it might involve extensive segmental resection or duodenopancreatectomy. With advances in endoscopic techniques, endoscopic mucosal resection (EMR) is being increasingly performed for the removal of duodenal polyps. However, due to their rare occurrence, more supportive evidence on and adverse events was required in $2 \%$ (95\% confidence interval $[\mathrm{CI}] 0-4 \%$ ). EMR was successfully accomplished in $93 \%$ (95\%CI $89-97 \%$ ). The overall bleeding rate after EMR was 16\% (95\%Cl $10-23 \%)$, and the pooled delayed bleeding rate was $5 \%(95 \% \mathrm{Cl} 2-7 \%)$. The overall incidence of perforation was $1 \%$ (95\%CI $1-3 \%$ ). Over a median follow-up period of 6-72 months, the recurrence rate after EMR was $15 \%$ (95\%CI 7-23\%). Six studies (pooled recurrence $20 \%$, 95\%CI 14 $27 \%$ ) reported on the outcomes of managing recurrent polyps, for which endoscopic removal was successful in $62 \%$ (95\%CI $37-87 \%)$. There was no procedure related mortality.

Conclusion: EMR appears to be a safe and effective therapeutic option for management of sporadic non-ampullary duodenal polyps. Longterm endoscopic surveillance is required to manage and treat recurrent disease.

the safety and efficacy of EMR of duodenal polyps, the frequency of follow-up, and management of recurrent disease is needed.

EMR seems to be a reasonable initial approach for sporadically occurring non-ampullary duodenal polyps to prevent their malignant transformation. A majority of published studies on EMR of duodenal polyps are retrospective and include a small patient cohort. To our knowledge, a systematic review of available evidence evaluating the efficacy and safety of EMR has not been published. Our main objective was to perform a systematic review and meta-analysis of studies on EMR of sporadic non-ampullary duodenal polyps to evaluate the safety and efficacy of the technique. 


\section{Methods}

$\nabla$

\section{Literature search}

We reviewed the PUBMED and EMBASE databases for studies published to May 2015. Search words included "Endoscopic resection and duodenal tumors", "Endoscopic resection and duodenal polyps", “Non-ampullary duodenal polyps", “Endoscopic Mucosal Resection", and "Endoscopic snare resection". Two reviewers (VL and $\mathrm{MH}$ ) independently reviewed the articles according to predefined inclusion and exclusion criteria. Any differences were resolved by mutual agreement and in consultation with the third reviewer (UN).

\section{Selection criteria}

Only studies employing EMR (with submucosal injection and/or underwater technique) of sporadic non-ampullary duodenal polyps were included. Studies with endoscopic submucosal dissection (ESD) or argon plasma coagulation (APC) alone as the major treatment modality were excluded from our analysis. Studies reporting the role of EMR in familial duodenal polyps were also excluded. Similarly, studies with a significant number of adenocarcinomas were also excluded as they are effectively managed by surgery rather than endoscopic methods. We included only duodenal polyps managed by EMR. We excluded patients who had ESD, or EMR performed through double balloon enteroscopy. Abstracts were also included according to the eligibility criteria. Case series with less than 10 patients were not included in the analysis.

\section{Quality of studies}

The quality of the included studies was assessed using the Newcastle-Ottawa scale (NOS). A maximum of 9 points were awarded on the basis of the cohort selection ( $<4$ points), the comparability of the cohort design and analysis ( $<2$ points), and the adequacy of outcome measures ( $<3$ points); $>6$ points was considered to be high quality. Supplementary Table 1 highlights the quality of studies based on NOS.

\section{Data availability}

Data on the success rates of EMR were available for all studies. Surgical intervention rates for the failure of EMR/recurrences and to manage perforation/significant post-procedural bleeding were available for all but one study. Information on overall bleeding rates and perforation was available for all studies. Two studies did not differentiate between intraprocedural and delayed bleed- ing rates. Details on recurrences were not available for two studies. Data on successful endoscopic management of recurrences were available for six studies.

\section{Outcome measurements}

The primary outcome measure was the surgical intervention rates due to non-curative endoscopic resection (incomplete removal/recurrence necessitating surgery) and surgery for managing adverse events. We adopted this outcome from a recent systematic review on colon EMR [5]. Secondary outcomes included (a) the rate of complete endoscopic resection of the polyps, (b) adverse events (bleeding-intraprocedural and delayed, and perforation), (c) long-term polyp recurrence and successful endoscopic management of recurrences, (d) malignant transformation, and (e) mortality. Surgical intervention rates are exclusive of patients who directly underwent surgery before any attempt at initial endoscopic resection.

\section{Statistical analysis}

This meta-analysis was performed by calculating pooled proportions. First, the individual study proportion was transformed into a quantity using the Freeman - Tukey variant of the arcsine square root transformed proportion. The pooled proportion was calculated as the back-transform of the weighted mean of the transformed proportions, using inverse arcsine variance weights for the fixed effects model and DerSimonian - Laird weights for the random effects model. Forest plots were constructed to show the point estimates in each study in relation to the summary pooled estimate. Prediction interval was estimated. The width of the point estimates in the Forest plots corresponded to the assigned weight of the study. Heterogeneity among the studies was assessed using Cochran's "Q" test (with tau ${ }^{2}$ and a $P$-value) and the $I^{2}$ statistics where values less than $25 \%$ indicated low heterogeneity. A random-effects model was used when the heterogeneity was high $\left(I^{2}>75 \%\right)$. Combined weighted proportions and meta-regression were determined using STATA version 13 (STATA Corp LP, College Station, Texas, United States). The robustness of the meta-analysis to publication bias was assessed by funnel plots and bias indicators, including the Harbord-Egger test. The small study effect and its impact on publication bias was also evaluated by cumulative meta-analysis (cumulatively adding studies according to sample size of primary studies - from largest to smallest sample size). The study data are arranged from the largest to the smallest study, and meta-analyses were run in a stepwise fashion.

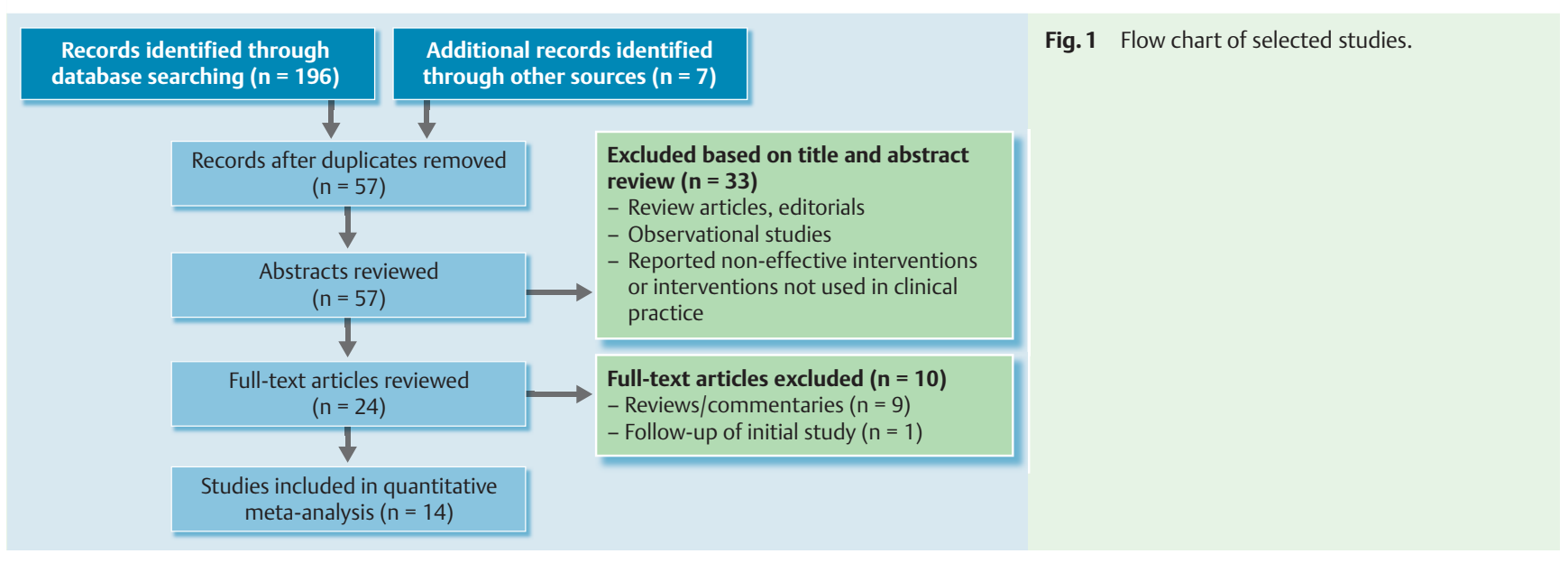




\section{Results}

$\nabla$

The initial screening using our search criteria yielded 196 studies. From these studies, 57 articles were retrieved and reviewed. - Fig. 1 shows the flow diagram for study selection. Finally, 14 studies fulfilling the eligibility criteria were included for our systematic review [6-19].

A total of 14 studies included 440 patients of which $48.5 \%$ were males. There were a total of 485 duodenal polyps which were removed by EMR, and adenomas accounted for the majority of treated lesions (90\%) with adenocarcinomas constituting only $1.9 \%$. The mean size of the polyps in the 14 studies ranged from $13 \mathrm{~mm}$ to $35 \mathrm{~mm}$. From the studies with available data, the majority of the polyps were sessile (92\%) and located in the second part of the duodenum (79.8\%). Tubular adenomas (52\%) were the most common histological lesion followed by villous/tubulovillous adenomas (48\%). Among the lesions where EMR was performed, $45 \%$ of the lesions were resected en bloc and $55 \%$ by piecemeal technique. Adjuvant APC post-EMR was employed in $29 \%$ of the polypectomy procedures to achieve complete resection. From the available data, $90 \%$ of polyps could be removed completely at the initial endoscopy, and the remaining $10 \%$ required multiple sessions $(2-3)$ to achieve initial complete eradication. - Table 1 details the demographic characteristics and the clinical information on the polyps and outcomes of EMR in the various studies.

\section{Primary end point}

A total of $12 / 402$ (3\%) patients underwent surgery after initial EMR. Surgery was performed in 8/402 patients for non-curative EMR, of which three had developed malignant transformation; 4/402 patients underwent surgery for management of procedure related adverse events ( 3 perforations, 1 hemorrhage). The overall surgical intervention rates among the studies was $2 \%(95 \% \mathrm{CI}$ $\left.0-4 \%, I^{2}=0\right)(\bullet$ Fig. 2 ).

\section{Secondary end points}

Successful complete removal of duodenal polyps using EMR was accomplished in 93\% (95\%CI 89-97\%) irrespective of long-term recurrences or the need for surgery $(\bullet$ Fig. 3 ). The pooled proportion of overall bleeding was $16 \%$ (95\%CI 10-23\%) ( Fig.4). Twelve studies differentiated delayed bleeding from intraprocedural bleeding; delayed bleeding following EMR was 5\% $(95 \% \mathrm{Cl}$ $2-7 \%$ ) ( Fig.5). Endoscopic perforation occurred in 1\% (95\% $\mathrm{CI}-1 \%$ to $3 \%$ ) ( Fig.6). While all intraprocedural perforations were managed endoscopically, delayed perforations required surgery. There was a fatal case of perforation involving a 76year-old man who died 2 months after surgical intervention due to a cerebrovascular event which was not a direct consequence of the procedure. There were no other reported mortalities directly related to EMR.

\section{Follow-up}

The median follow-up period was $6-72$ months, and the pooled recurrence of the polyps during this follow-up was $15 \%(95 \% \mathrm{CI}$ $7-23 \%$ ) ( Fig.7). Six studies (pooled recurrence 20\%, 95\%Cl $14-27 \%$ ) reported the outcomes of managing those recurrent polyps, and successful endoscopic removal was accomplished in $62 \%$ (95\%CI $37-87 \%$ ) ( Fig. 8). Malignant transformation of the adenomas was observed in three recurrent cases, and all three were referred for surgical management. There was a significant increase in the odds of recurrence of villous/tubulovillous adenomas compared to tubular adenomas (OR 4.86, 95\%CI 1.62-14.63) (๑ Fig.9).

\section{Publication bias}

We evaluated the possibility of publication bias for the surgical intervention rates using a funnel plot and Egger's regression test. Both of these methods showed that small study publication bias was marginally not significant $(P=0.052)$ if $\alpha$ was set at $<0.05$ ( Supplementary Fig. 1 ). However, with a conservative level of $\alpha<0.1$, the small study publication bias was rendered as being significant in this systematic review. To further investigate the publication bias, we conducted a cumulative meta-a-

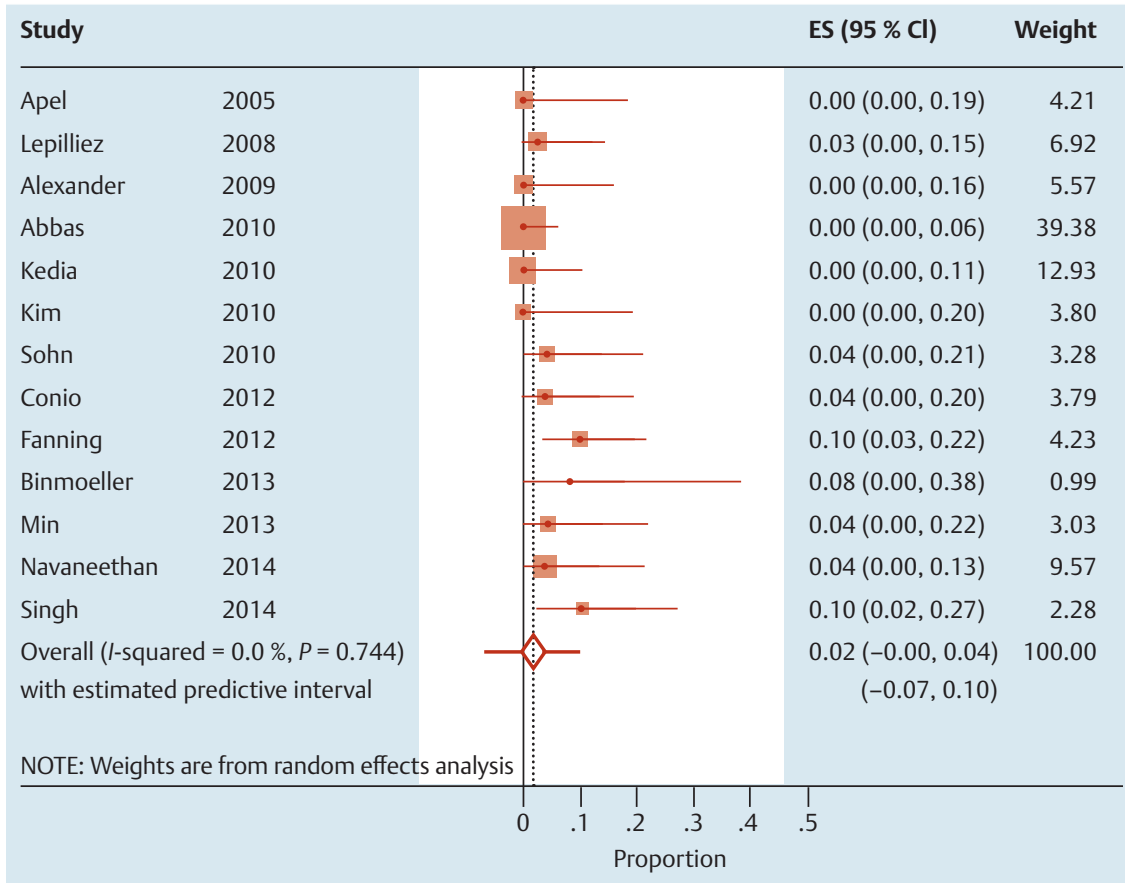

Fig. 2 Forrest plot of studies reporting surgical intervention rates. The pooled surgical intervention rate was $2 \%(95 \% \mathrm{Cl} 0-4 \%)$. 

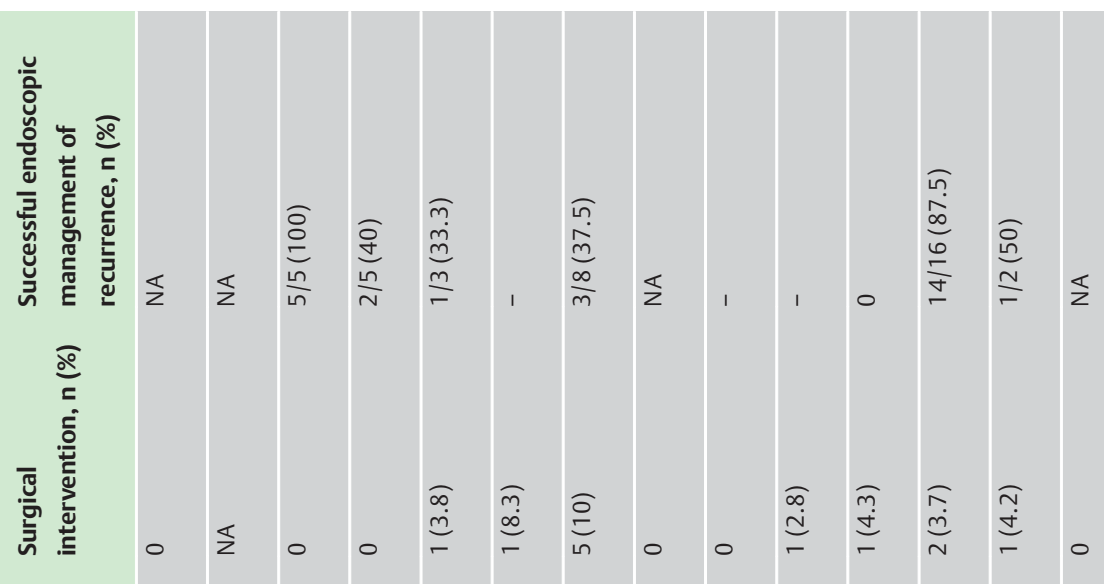

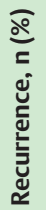

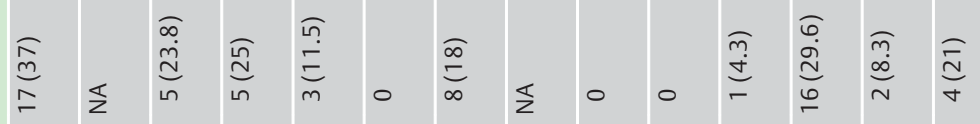

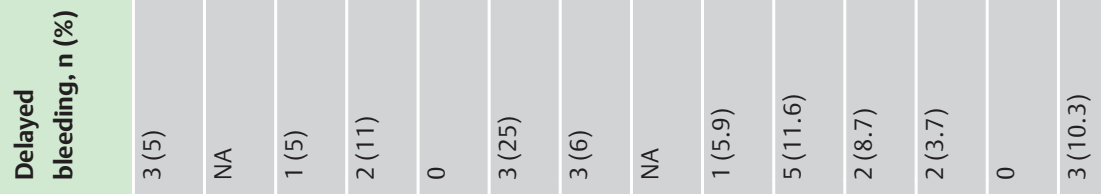

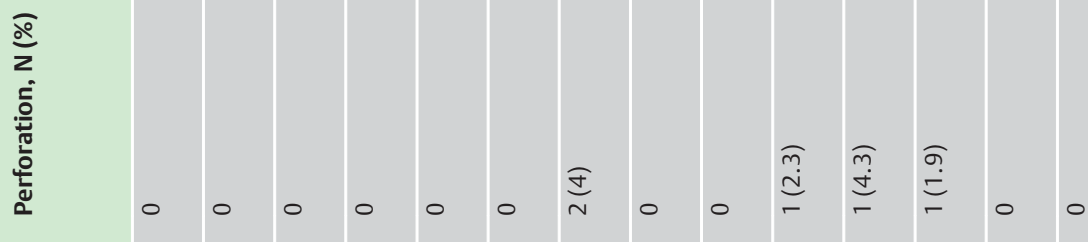

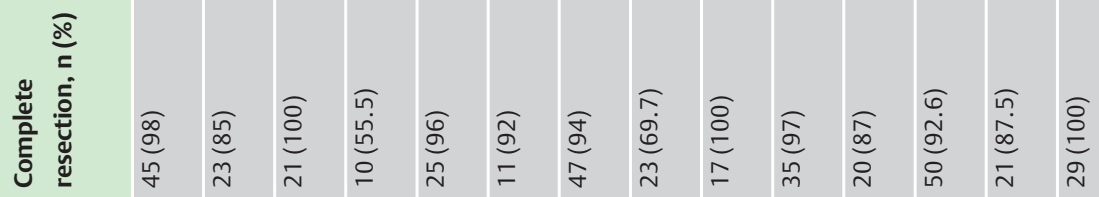

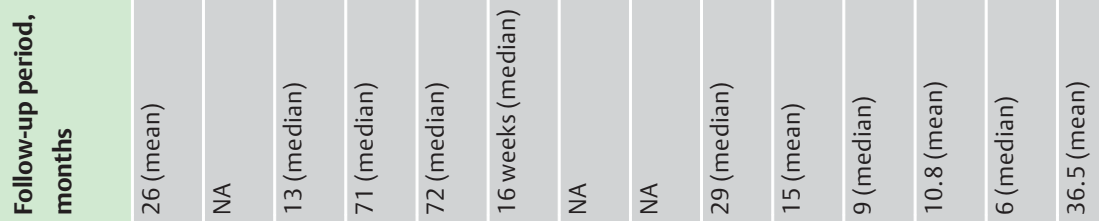

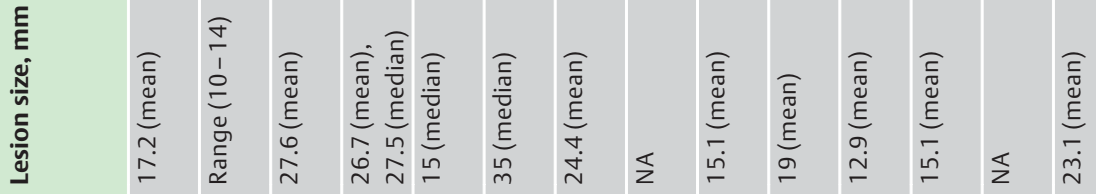

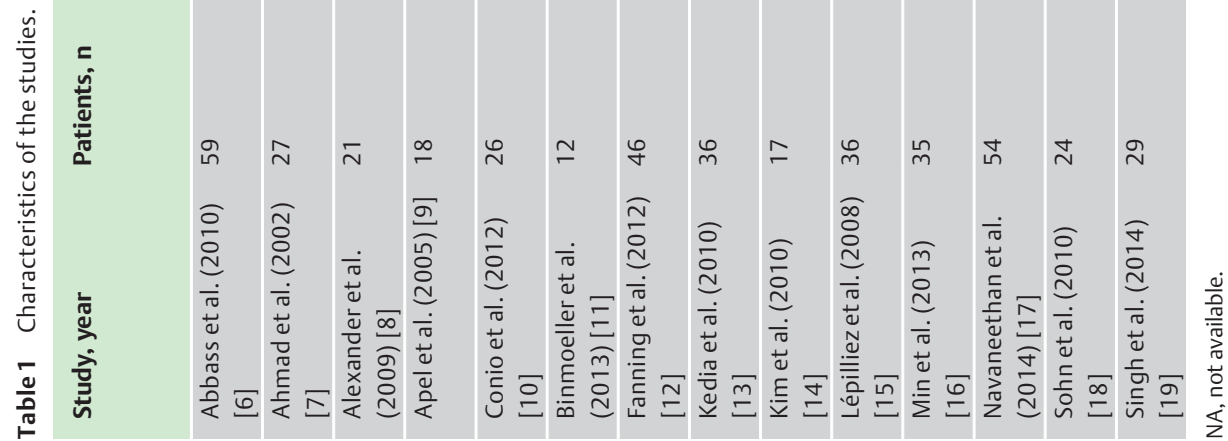




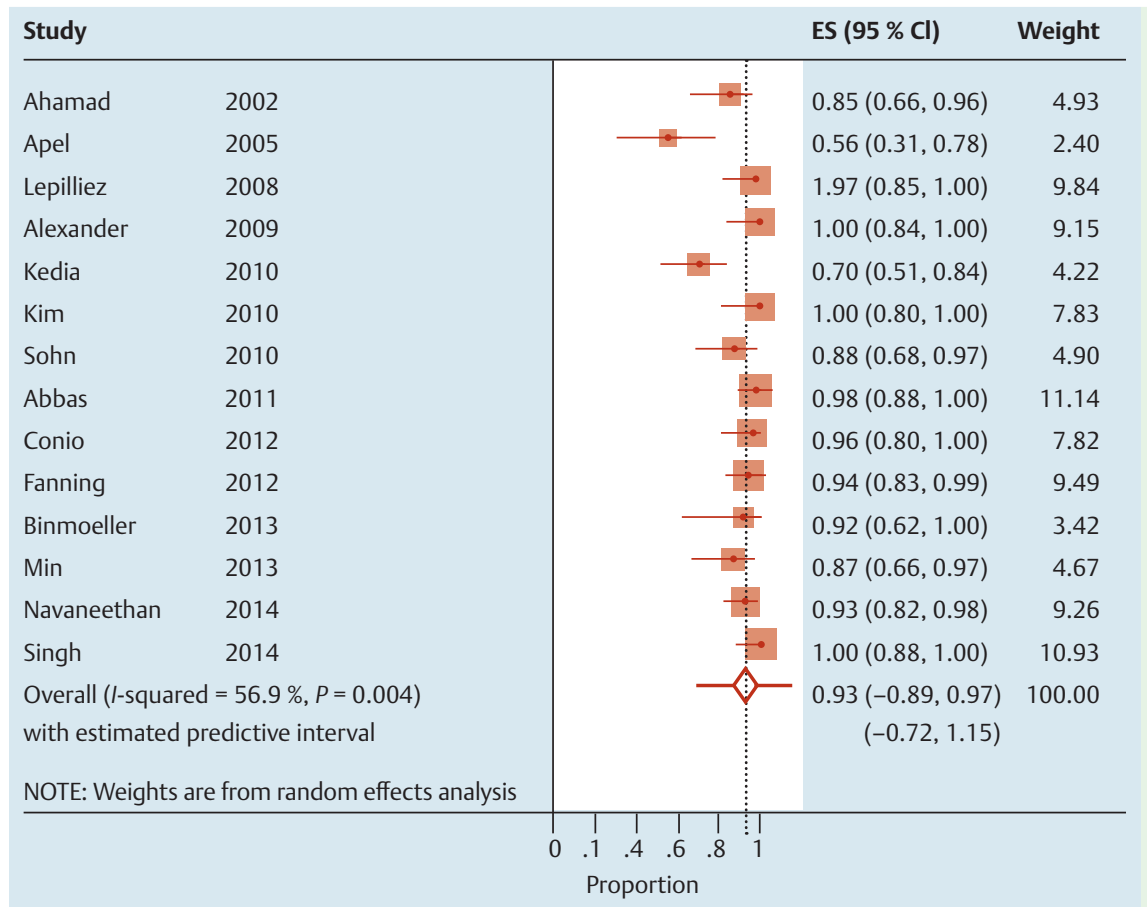

Fig. 3 Forrest plot of studies reporting complete endoscopic removal rates. The pooled complete endoscopic removal rate was $93 \%(95 \% \mathrm{Cl} 89$ $97 \%)$.

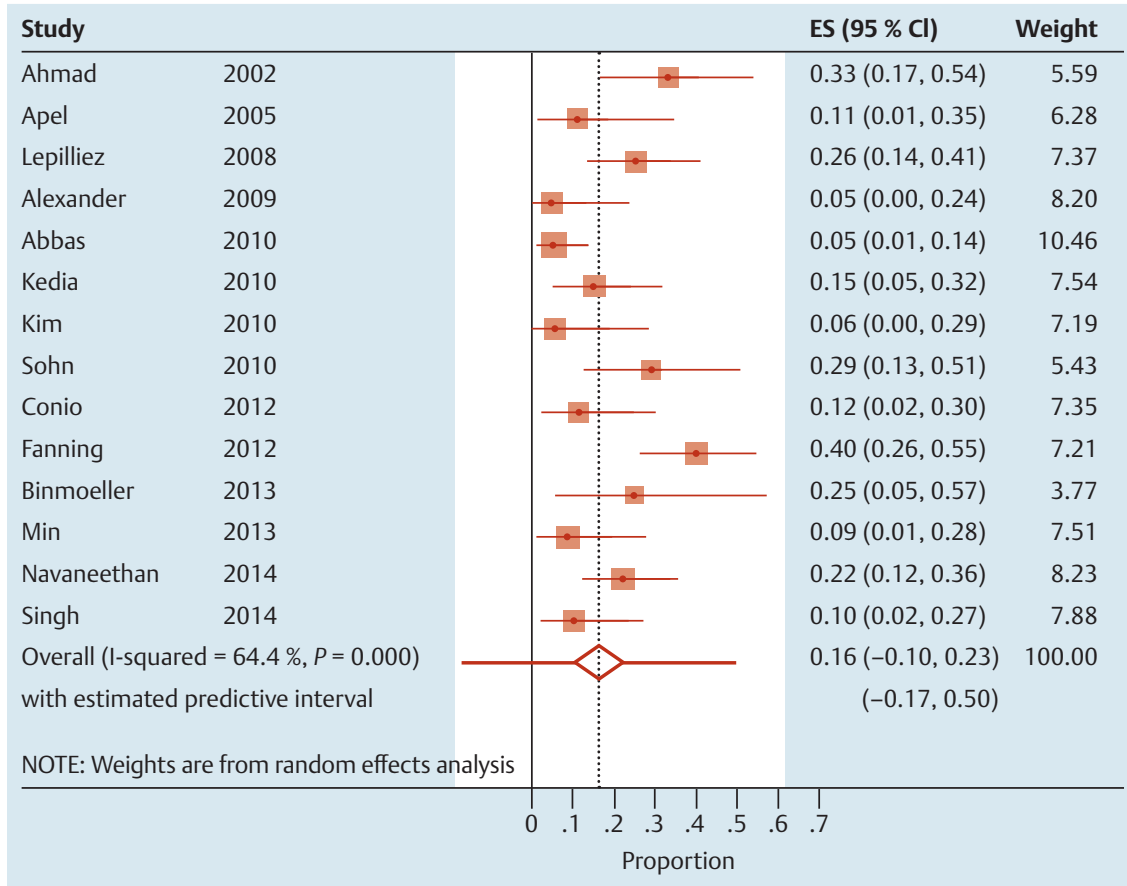

Fig.4 Forrest plot of studies reporting the proportion of overall bleeding. The pooled proportion of overall bleeding was $16 \%(95 \% \mathrm{Cl} 10-23 \%)$. nalysis. In the cumulative meta-analysis, all of the studies were sorted by sample size in the sequence of the largest to smallest. We iterated the meta-analysis by adding one additional study each time. This resulted in a series of cumulative mean estimates, each based on one more effect size than the previous mean ( Supplementary Fig.2). The figure demonstrates that by including six large studies, with a cumulative sample size of 261 patients, this yielded a mean surgical intervention rate of $2.4 \%$ (95\%CI-0.6\% to 5.3\%). As smaller studies were added, the overall surgical intervention rate drifted slightly lower to $1.9 \%$ ( $95 \% \mathrm{CI}-0.2 \%$ to $4.0 \%)$. The point estimate and its confidence levels did not shift with the addition of the remaining four smaller studies suggesting that the publication bias was not significant in our study.

\section{Discussion}

Sporadic non-ampullary duodenal polyps are being increasingly identified with the more widespread use of upper endoscopy. Surgery to remove these polyps carries high rates of morbidity and mortality. From our analysis, EMR of duodenal polyps appears to be a safe and effective alternative to surgery, with a very low need for surgical intervention ( $2 \%)$. The polyp recur- 


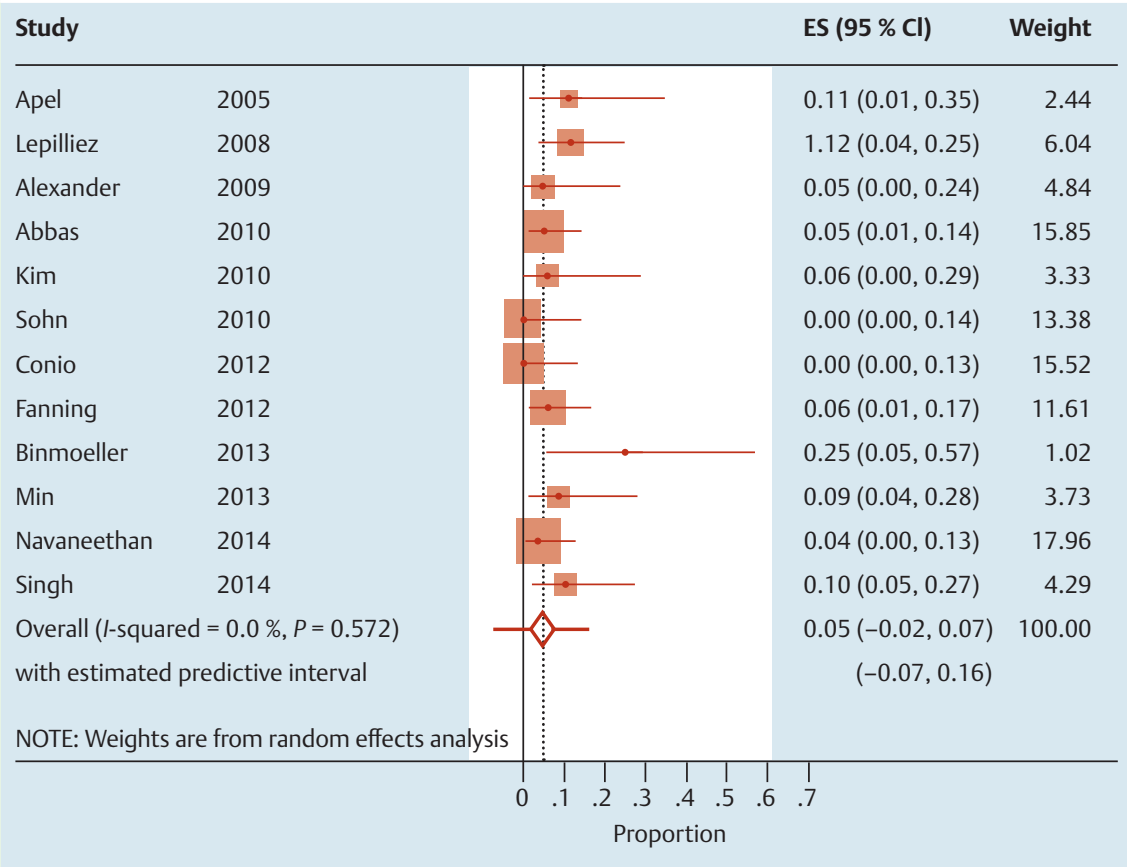

Fig. 5 Forrest plot of studies reporting the proportion of delayed bleeding following EMR. The pooled proportion of delayed bleeding following EMR was $5 \%$ ( $95 \% \mathrm{Cl} 2-7 \%)$.

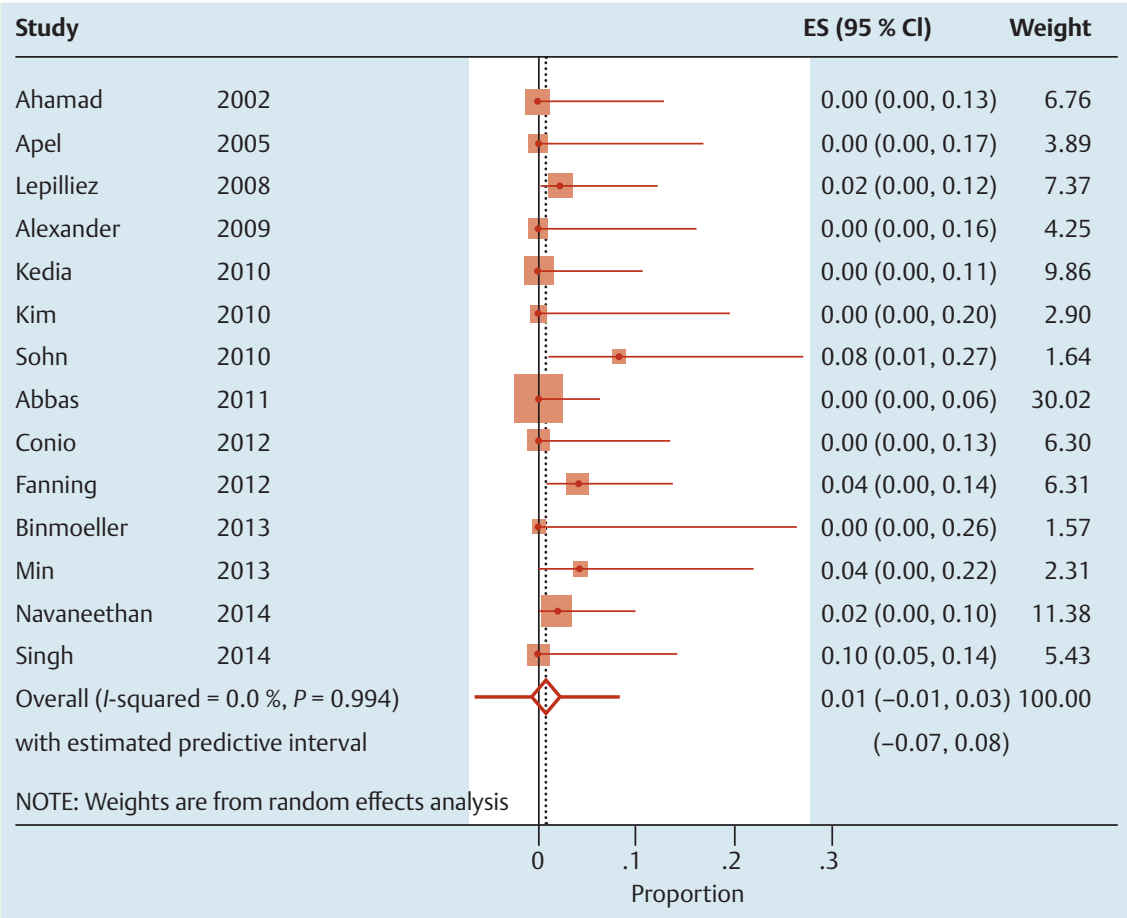

Fig. 6 Forrest plot of studies reporting the proportion of perforation. The pooled rate of endoscopic perforation was $1 \%(95 \% \mathrm{Cl}-1 \%$ to $3 \%)$. rence rate was $15 \%$, the majority of them could be managed endoscopically on long-term follow-up, and the rate of malignant transformation was very low. The very low rate of surgical intervention, low rate of adverse events, and no cases of mortality directly related to polypectomy further emphasize that EMR of duodenal polyps can be undertaken safely with high efficacy.

Submucosal injection provides the necessary cushion for polyp removal as most lesions are sessile. Underwater EMR has a similar principle and separates the mucosa and submucosa away from the deeper muscularis propria layer and allows EMR. EMR is effective in achieving complete polyp removal as evidenced by a pooled overall complete resection rate of $93 \%$. EMR of duodenal polyps can be challenging due to the thin wall and rich vascularity of the duodenum [20]. This raises concern with regard to the possibility of perforation and hemorrhage. An increased risk of perforation has been observed in studies involving endoscopic submucosal dissection of duodenal polyps [21,22]. From our analysis, the low perforation rates reinforce the view that EMR can be done safely for the removal of duodenal polyps, especially with submucosal lift which can practically increase the thickness of the duodenal wall facilitating safer removal of the polyps.

The pooled delayed bleeding rate in our meta-analysis is $5 \%$ which appears reasonable. Most of the cases of delayed bleeding were managed endoscopically, with only one case requiring sur- 


\begin{tabular}{|c|c|c|c|c|c|}
\hline \multicolumn{4}{|l|}{ Study } & ES (95 \% Cl) & Weight \\
\hline Apel & 2005 & & $\rightarrow$ & $0.25(0.09,0.49)$ & 6.97 \\
\hline Lepilliez & 2008 & $\frac{1}{1}$ & & $0.00(0.00,0.10)$ & 11.85 \\
\hline Alexander & 2009 & & $\cdot$ & $0.24(0.08,0.47)$ & 7.20 \\
\hline Abbas & 2010 & & $\rightarrow$ & $0.38(0.24,0.53)$ & 8.73 \\
\hline Kim & 2010 & $\frac{1}{10}$ & & $0.00(0.00,0.20)$ & 10.49 \\
\hline Sohn & 2010 & $\rightarrow$ & & $0.08(0.01,0.27)$ & 9.38 \\
\hline Conio & 2012 & 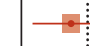 & & $0.12(0.02,0.30)$ & 9.08 \\
\hline Fanning & 2012 & & - & $0.18(0.08,0.33)$ & 9.64 \\
\hline Min & 2013 & + & & $0.04(0.00,0.22)$ & 10.10 \\
\hline Navaneethan & 2014 & & $\longrightarrow$ & $0.30(0.18,0.44)$ & 9.44 \\
\hline Singh & 2014 & & - & $0.21(0.09,0.46)$ & 7.12 \\
\hline \multicolumn{2}{|c|}{$\begin{array}{l}\text { Overall }(I-\text { squared }=79.8 \%, P=0.000) \\
\text { with estimated predictive interval }\end{array}$} & & & \multicolumn{2}{|c|}{$\begin{array}{c}0.15(-0.07,0.23) 100.00 \\
(-0.31,0.61)\end{array}$} \\
\hline \multicolumn{2}{|c|}{ NOTE: Weights are from random effects analysis } & & & & \\
\hline
\end{tabular}

Fig. 7 Forrest plot of studies reporting the pooled recurrence of the polyps following EMR. The pooled recurrence rate was $15 \%(95 \% \mathrm{Cl} 7-23 \%)$.

\section{Study}

\begin{tabular}{|c|c|c|c|c|}
\hline Study & & & ES (95 \% Cl) & Weight \\
\hline Apel & 2005 & $\rightarrow$ & $0.40(0.05,0.85)$ & 15.08 \\
\hline Alexander & 2009 & & $1.00(0.48,1.00)$ & 19.53 \\
\hline Sohn & 2010 & $\longrightarrow$ & $0.50(0.01,0.88)$ & 12.66 \\
\hline Conio & 2012 & $\rightarrow$ & $0.33(0.01,0.91)$ & 13.69 \\
\hline Fanning & 2012 & $\rightarrow$ & $0.38(0.09,0.76)$ & 17.11 \\
\hline Navaneethan & 2014 & & $0.88(0.62,0.98)$ & 21.94 \\
\hline Overall (I-squa & $=71.1 \%, P=0.004)$ & & $0.62(0.37,0.87)$ & 100.00 \\
\hline with estimate & dictive interval & & $(-0.95,1.19)$ & \\
\hline NOTE: Weight & from random effects analysis & & & \\
\hline & & 0.2 .4 & & \\
\hline & & Prop & & \\
\hline
\end{tabular}

Fig. 8 Forrest plot of studies reporting the endoscopic treatment of recurrent polyps. Successful endoscopic removal was accomplished in $62 \%$ ( $95 \%$ (Cl 37-87\%).

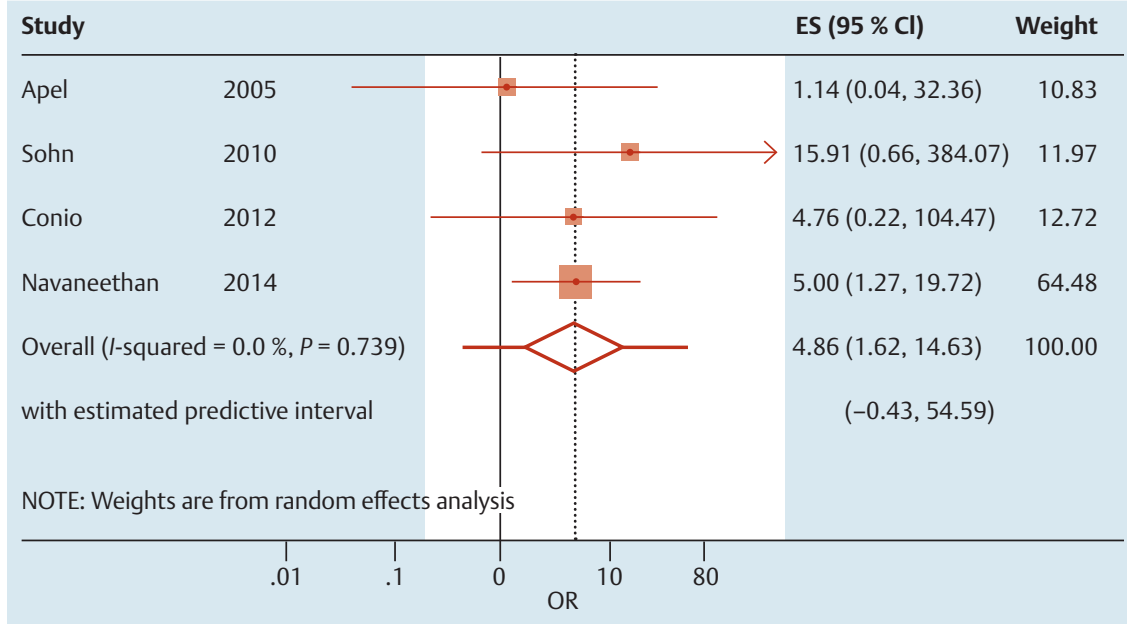

Fig.9 Forrest plot of studies reporting the recurrence rate of tubular adenomas vs. villous/tubulovillous adenomas. There was an increase in the odds of recurrence of villous/tubulovillous adenomas compared to tubular adenomas (OR $4.86,95 \% \mathrm{Cl}$ $1.62-14.63)$. 
gical intervention. A delayed bleeding rate of $25 \%$ was observed in one study in which the reported median size of the polyps was $35 \mathrm{~mm}$ [12]. In the same study, intraprocedural bleeding occurred significantly more frequently in polyps that were larger than $30 \mathrm{~mm}$ compared to smaller lesions ( $57.8 \%$ vs. $19.3 \%, P=$ 0.005). In another study, no delayed bleeding was observed when clipping or APC was performed compared with increased bleeding episodes in $22 \%$ of the procedures where these measures were not undertaken [15]. Although ablation techniques and endoscopic clips can possibly reduce delayed bleeding, these factors could not be analyzed due to the lack of sufficient comparable data. Low rates of perforation (comparable to that of colonic polypectomy) and delayed bleeding rates coupled with less need for surgery due to adverse events justify the safety of the EMR technique [23].

The pooled recurrence rate of polyps after initial EMR was 15\%, and many of the recurrent polyps (data on removing recurrent polyps were provided in six studies) could be treated successfully by endoscopic techniques or the use of APC. Recurrences were more common with villous lesions [17], that are associated with increased rates of recurrence and malignant transformations, and in most cases require surgery $[3,24]$. When this association was analyzed in the studies where sufficient data were available, we found significantly increased odds of recurrence for villous/ tubulovillous adenomas. Villous/tubulovillous adenomas merit continued and careful surveillance even after complete resection. There was a lack of sufficient data on the management technique of recurrent polyps, but most studies deployed a combination of snare/APC for the management of recurrences. Among the factors analyzed for the recurrence of the polyps, only the villous nature of the polyps was associated with increased recurrence. The risk of recurrence did not differ based on the initial type of polypectomy-en bloc or piecemeal. Additional thermal methods after snare polypectomy, especially in villous adenomas, were suggested by Apel et al. [9] that could decrease the rate of polyp recurrences. In the study by Kedia et al. [13], complete eradication was more likely if the polyps involved less than $50 \%$ of the mucosal surface. The factors that could impact the risk of recurrence including size of the polyps/circumference of the lesion, percentage of polyp removal by piecemeal technique/adjuvant APC/ thermal ablation are beyond the scope of this meta-analysis, and need to be determined in future large prospective studies.

The timing and frequencies of surveillance endoscopies differed among the studies. Though it is difficult to formulate evidencebased guidelines for follow-up, the ideal recommendation would be 3 months from the initial EMR to identify and treat any residual lesions and/or early recurrence irrespective of the histology or size of the lesion. Tubular adenomas can then be followed yearly for the first 2 years and then once every $2-3$ years which appears reasonable. Polyps with tubulovillous/villous histology may require a more frequent follow-up since the propensity for increased recurrence and hence surveillance in these patients need to be individualized.

Our study has limitations. Though there was no heterogeneity in the primary end point of our analysis, there was a considerable heterogeneity in the recurrence of polyps. All of the studies included were retrospective analyses involving a small patient cohort. The procedural techniques were not standardized and the application of endoscopic clips or ablation methods after polypectomy varied amongst the studies. The eventual need for surgery implies failure of the EMR technique, and hence was considered to be the primary outcome measure of our study. It also re- flects the safety of the procedure providing information on serious adverse events that is more meaningful than the overall adverse events for determining procedural safety. However, evaluating endoscopic recurrence rate as the primary outcome measure has its demerits. Universal histological confirmation of absence of recurrence was not performed in the included studies, and hence it may not be a true measure of recurrence. Also the definition of recurrence was not unanimous in all studies, and residual lesions could have been included as recurrences as many of the surveillance endoscopies did not start from the first negative follow-up endoscopy. Therefore, as a measure of both the procedural efficacy and safety, surgical intervention rate appears to be a better primary outcome measure than evaluation of endoscopic recurrence. Finally, none of the studies compared the procedural costs or outcomes with surgery.

Given the high rate of complete endoscopic resection, less need for salvage surgery, low rates of procedure-related adverse events, and the ability to treat the majority of recurrences effectively, EMR must be considered to be an effective alternative to surgery. Long-term follow-up appears critical, particularly for villous and tubulovillous polyps.

Competing interests: Robert Hawes is a consultant for Olympus and Boston Scientific. Shyam Varadarajulu is a consultant for Boston Scientific and Olympus. Udayakumar Navaneethan is a consultant for AbbVie, Janssen and Takeda. None of the other authors declared a financial conflict of interest.

\section{References}

1 Culver EL, McIntyre AS. Sporadic duodenal polyps: classification, investigation, and management. Endoscopy 2011; 43: 144-155

2 Basford PJ, Bhandari P. Endoscopic management of nonampullary duodenal polyps. Ther Adv Gastroenterol 2012; 5: 127-138

3 Galandiuk S, Hermann RE, Jagelman DG et al. Villous tumours of the duodenum. Ann Surg 1988; 207: 234-239

4 Van Heumen BW, Mul K, Nagtegaal ID et al. Management of sporadic duodenal adenomas and the association with colorectal neoplasms: a retrospective cohort study. J Clin Gastroenterol 2012; 46: 390 - 396

5 Hassan C, Repici A, Sharma P et al. Efficacy and safety of endoscopic resection of large colorectal polyps: a systematic review and meta-analysis. Gut 2016; 65: 806-820

6 Abbass R, Rigaux J, Al-Kawas FH. Nonampullary duodenal polyps: characteristics and endoscopic management. Gastrointest Endosc 2010; 71: $754-759$

7 Ahmad NA, Kochman ML, Long WB et al. Efficacy, safety, and clinical outcomes of endoscopic mucosal resection: a study of 101 cases. Gastrointest Endosc 2002; 55: 390-396

8 Alexander S, Bourke MJ, Williams SJ et al. EMR of large, sessile, sporadic nonampullary duodenal adenomas: technical aspects and long-term outcome (with videos). Gastrointest Endosc 2009; 69: 66-73

9 Apel D, Jakobs R, Spiethoff $A$ et al. Follow-up after endoscopic snare resection of duodenal adenomas. Endoscopy 2005; 37: $444-448$

10 Conio M, De Ceglie A, Filiberti R et al. Cap-assisted EMR of large, sporadic, nonampullary duodenal polyps. Gastrointest Endosc 2012; 76: $1160-1169$

11 Binmoeller KF, Shah JN, Bhat YM et al. "Underwater" EMR of sporadic laterally spreading nonampullary duodenal adenomas (with video). Gastrointest Endosc 2013; 78: 496-502

12 Fanning $S B$, Bourke MJ, Williams SJ et al. Giant laterally spreading tumors of the duodenum: endoscopic resection outcomes, limitations, and caveats. Gastrointest Endosc 2012; 75: 805-812

13 Kedia P, Brensinger C, Ginsberg G. Endoscopic predictors of successful endoluminal eradication in sporadic duodenal adenomas and its acute complications. Gastrointest Endosc 2010; 72: 1297-1301

14 Kim HK, Chung WC, Lee BI et al. Efficacy and long-term outcome of endoscopic treatment of sporadic nonampullary duodenal adenoma. Gut Liver 2010; 4: 373-377 
15 Lépilliez V, Chemaly M, Ponchon $T$ et al. Endoscopic resection of sporadic duodenal adenomas: an efficient technique with a substantial risk of delayed bleeding. Endoscopy 2008; 40: 806 -810

16 Min YW, Min BH, Kim ER et al. Efficacy and safety of endoscopic treatment for nonampullary sporadic duodenal adenomas. Dig Dis Sci 2013; $58: 2926-2932$

17 Navaneethan $U$, Lourdusamy D, Mehta $D$ et al. Endoscopic resection of large sporadic non-ampullary duodenal polyps: efficacy and longterm recurrence. Surg Endosc 2014; 28: 2616-2622

18 Sohn JW, Jeon SW, Cho CM et al. Endoscopic resection of duodenal neoplasms: a single-center study. Surg Endosc 2010; 24: 3195-3200

19 Singh A, Siddiqui U, Konda V et al. Efficacy and safety of endoscopic mucosal resection for large non-ampullary duodenal adenomas: a single U.S. center experience. American College of Gastroenterology Abstract 2014

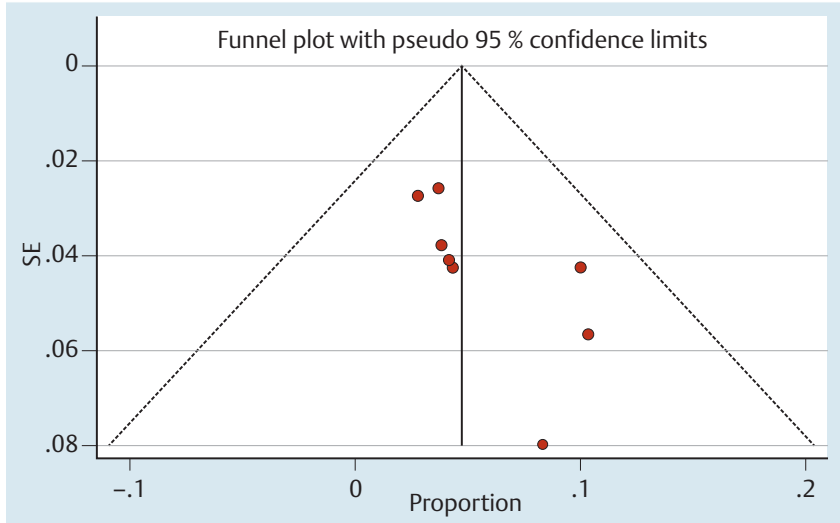

Supplementary Fig. 1 Funnel plot of data included in this meta-analysis (against their standard errors)
20 Bourke MJ. Endoscopic resection in the duodenum: current limitations and future directions. Endoscopy 2013; 45: 127-132

21 Honda T, Yamamoto H, Osawa $H$ et al. Endoscopic submucosal dissection for superficial duodenal neoplasms. Dig Endosc 2009; 21: 270 274

22 Takahashi T, Ando T, Kabeshima $Y$ et al. Borderline cases between benignancy and malignancy of the duodenum diagnosed successfully by endoscopic submucosal dissection. Scand J Gastroenterol 2009; 44: $1377-1383$

23 Kedia P, Waye JD. Colon polypectomy: a review of routine and advanced techniques. J Clin Gastroenterol 2013; 47: 657-665

24 Farnell MB, Sakorafas GH, Sarr MG et al. Villous tumors of the duodenum: reappraisal of local vs. extended resection. J Gastrointest Surg 2000; 4 : $13-21$

\begin{tabular}{|c|c|c|c|}
\hline \multicolumn{3}{|l|}{ Study } & \multirow{2}{*}{$\begin{array}{l}\text { ES }(95 \% \text { Cl) } \\
0.000(-0.030,0.030)\end{array}$} \\
\hline Abbas & 2010 & & \\
\hline + Navaneethan & 2014 & & $0.008(-0.022,0.039)$ \\
\hline + Fanning & 2012 & & $0.032(-0.019,0.083)$ \\
\hline + Lepilliez & 2008 & & $0.026(-0.010,0.063)$ \\
\hline + Kedia & 2010 & & $0.017(-0.010,0.045)$ \\
\hline+ Singh & 2014 & & $0.024(-0.006,0.053)$ \\
\hline+ Conio & 2012 & & $0.022(-0.004,0.048)$ \\
\hline + Sohn & 2010 & & $0.020(-0.002,0.042)$ \\
\hline+ Min & 2013 & & $0.019(-0.002,0.040)$ \\
\hline + Alexander & 2009 & & $0.018(-0.002,0.038)$ \\
\hline + Apel & 2005 & & $0.017(-0.002,0.037)$ \\
\hline$+\mathrm{Kim}$ & 2010 & & $0.016(-0.003,0.035)$ \\
\hline + Binmoeller & 2013 & & $0.017(-0.002,0.036)$ \\
\hline & & $\begin{array}{cccc}.02 & .04 & .06 & .08 \\
\text { Proportion } & .08\end{array}$ & 1 \\
\hline
\end{tabular}

Supplementary Fig. 2 Results of a cumulative meta-analysis of surgical intervention data in which studies are sorted by size (largest to smallest). The first row was a meta-analysis based on the largest study (Abbas et al., $\mathrm{N}=59$ patients). The second row was a meta-analysis based on the first two studies (Abbas et al., $\mathrm{N}=59$ patients and Navaneethan et al., $N=54$ patients), and so on. The figure demonstrates that by including the six large studies, with a cumulative sample size of 261 patients, this yielded a mean for surgical intervention rate of $2.4 \%$ (95\% $\mathrm{Cl}-0.6 \%$ to $5.3 \%$ ). As smaller studies were added, the overall surgical intervention rate drifted slightly lower to $1.9 \%(95 \% \mathrm{Cl}-0.2 \%$ to $4.0 \%)$. The point estimate and its confidence levels did not shift with the addition of the remaining four smaller studies suggesting that the publication bias was not significant in our study. 


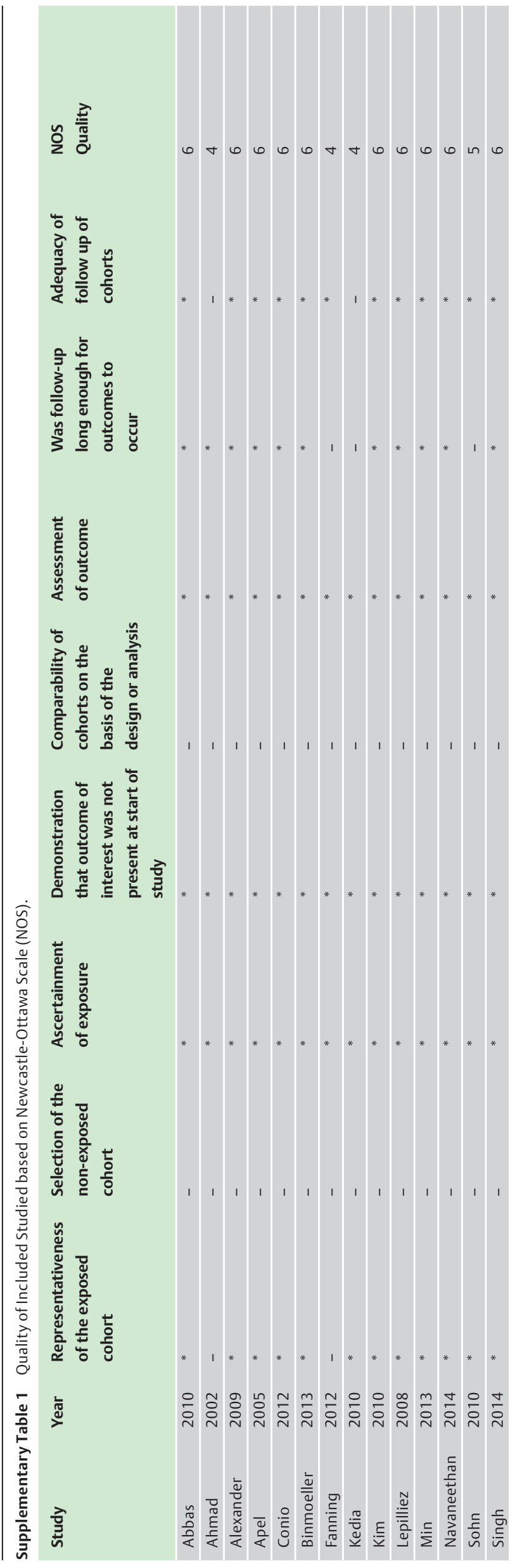

